\title{
Efficient Automated Liquid Detection in Microplates
}

\author{
Stefan Schinwald \\ Salzburg Univ. of \\ Applied Sciences \\ Urstein Sued 1, Salzburg \\ Austria \\ sschinwald.its-b2008@fh-salzburg.ac.at
}

\author{
Dominik Engel \\ Salzburg Univ. of \\ Applied Sciences \\ Urstein Sued 1, Salzburg \\ Austria \\ dominik.engel@fh-salzburg.ac.at
}

\author{
Maria Seidler \\ Tecan Austria \\ Untersbergstrasse 1a, \\ Groedig \\ Austria \\ maria.seidler@tecan.com
}

\begin{abstract}
An efficient approach to detecting the presence of liquid in microplates with a standard camera is proposed. The method is based on a modified Hough transformation followed by histogram-based thresholding. In comparison to previously suggested approaches, this method is computationally inexpensive. Effectiveness is evaluated by an empirical test setup.
\end{abstract}

\section{Introduction}

Microplates are used in a variety of standard molecular applications. A microplate consists of a number of so-called "wells" or "cavities", each of which contains a sample to be analyzed. A typical example of a molecular application using microplates is the Enzyme Linked ImmunoSorbent Assay (ELISA). Today's ELISA tests employ microplate washers, and microplate absorbance readers for the detection of viral pathogens. Many viral infections including the Hepatitis strains and HIV show no symptoms during first stages of infection. It is important to have effective high throughput screening methods to prevent transmission.

A quick and cost effective method for liquid detection in microplates for applications like the ELISA has the potential to enhance the quality of the process control and increase the general screening productivity. In particular, it is important to monitor the volume of liquid during the wash process to ensure correct sample preparation and the ensuing viral detection. Two central steps of the wash process include aspiration and dispensation of liquids and serums. A water detection algorithm could be implemented in the software to monitor the presence of liquid after dispensation and the absence of residual liquid volume after aspiration.

Should a faulty condition related to liquid level be detected over a software interface, a skilled technician could intervene and adjust the process parameters without losing samples. Such a liquid detection algorithm has potential to contribute to the further automatization of diagnostic robotic applications.

We propose an approach to detecting presence and absence of liquid in microplates. An image of a microplate array, which is created by a standard camera, is segmented and liquid detection, for example water detection, is performed by histogram-based thresholding.

The rest of this paper is organized as follows. Section 2 gives an overview of the state of the art and positions the contribution in the research background. The proposed method is presented in Section 3. Section 4 introduces the experimental setup and discusses empirical results. Section 5 concludes with an outlook on future research.

\section{State of the Art}

The main approaches to liquid measurement in microplates in today's industrial applications are capacitive, pressure, and ultrasonic detection methods, e.g. [9]. Although these methods are successful in determining liquid levels, they involve extensive hardware, mechanical complexity, and costly mathematical computations compared with the proposed approach. Also, the accurate detection of water droplets remains a challenge. With regard to the automation of a simple method for the precise detection of liquids based on image processing techniques, few proposals exist. It is common practice to manually inspect microplate arrays for the presence of liquids in the laboratory.

Depending on the perspective, the approach proposed here can be categorized in a number of disciplines: (i) automatic visual inspection, (ii) image classification, and (iii) image segmentation.

The method certainly falls into the category of automatic visual inspection. An overview of classic methods up to 1993 in this field is given by [6]. There are more recent 
approaches in this field that combine visual inspection with current classification methods, e.g. [7]. A number of methods for liquid classification have been proposed, including embedded systems, cf. [4]. In the area of liquid detection, a number of proposals for filling level detection in industrial bottle production exist, e.g. [12]. An overview is given by [8]. Some of these approaches use similar methods as the methods employed here, e.g. the use of preprocessing through edge detection. However, the problem domain is not comparable to the medical setup of liquid detection in microplates.

The approach presented here can be also viewed as a classification method with a binary decision to distinguish two classes. In this respect there are a number of existing approaches, mainly utilizing known classification methods. For example, support vector machines (SVM) have been proposed to perform histogram-based image classification [1]. Fuzzy versions of multidimensional histograms to improve image retrieval and classification have been proposed by [11]. There are a number of proposals for ROI-based classification.

As the method proposed here employs histogram-based thresholding, it is, in its basic principle, akin to thresholding techniques that are used in image segmentation. An extensive overview of thresholding methods up to 2004, including quantitative performance evaluation, can be found in [10]. More recent approaches focus on multi-resolution histograms for segmentation, e.g. [3] in the domain of medical image segmentation.

The contribution of the method proposed here beyond the state of the art is a histogram-based liquid detection approach in a clearly defined application scenario, which is both reliable and affordable. The fact that it is inexpensive both in computational and monetary terms, makes it feasible for real-world use and makes it a candidate to replace manual inspection of microplates.

\section{Liquid Detection}

The method for distinguishing between empty and filled cavities is based on the light absorption properties of liquids. The method can be used on any type of liquid. In our experimental setup we use the method for the detection of water. The maximum absorption coefficient for water for wavelengths between $380 \mathrm{~nm}$ and $1100 \mathrm{~nm}$ is reached around 960nm [2]. Using light of this wavelength to illuminate the cavities will therefore lead to the best results to distinguish empty from filled cavities. High absorption indicates the presence of water, while low absorption indicates the absence of water. Note that for detecting liquids other than water, light of different wavelengths may produce the best results.

Affordable LEDs can produce light with a maximum

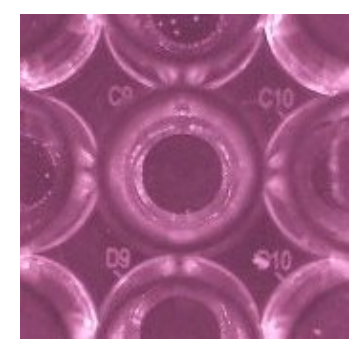

Figure 1. Example Microplate Input Image

wavelength of $950 \mathrm{~nm}$. To produce light beyond this wavelength, specialized laser equipment would be necessary. In our approach, which aims at an affordable solution, we therefore employ LEDs.

\subsection{Image Acquisition}

The proposed approach operates on images taken of each well by a camera from a position perpendicular to the microplate, as shown in Figure 1.

To capture the images a standard camera module is mounted on top of the microplate, as illustrated by Figure 2. By mounting a lens in front of the camera the large working distance is reduced. A transparent plastic ensures a homogenous illumination. This transparent plastic scatters the light by ten degrees. For illumination, an LED is mounted underneath the microplate.

\subsection{Modified Hough Algorithm for Cavity Localization}

A simplistic approach to determining liquid presence would be a simple histogram thresholding on the acquired image, without any cavity localization. However, our empirical evaluation of such an approach, given in Section 4, clearly shows that the level of detection accuracy is not acceptable in this case.

In order to achieve a level of accuracy that allows the method to be used in real life, our approach employs cavity localization before the thresholding step. Cavity localization restricts histogram processing to the region-of-interest only, i.e. the well located in the center of the input image. It is performed by edge detection followed by a modified Hough transformation. Although these steps add some computational complexity, they greatly contribute to accuracy by eliminating irrelevant input.

The variant of the Hough transform [5] used here is similar to Template Matching approaches. It searches for given structures in images. The searched structure has to be represented by edge pixels. The algorithm tries to find the edge 


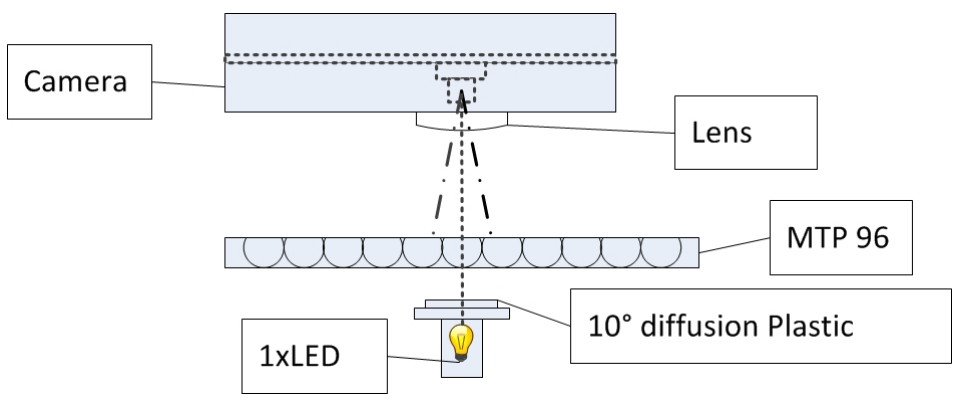

Figure 2. Image Acquisition

pixels which belong to the given geometric structure by employing a voting system: Every edge pixel votes for a structure it might belong to. To find a certain structure, it has to be brought down to its parameters. As an example for circles:

$$
\left(x-x_{0}\right)^{2}+\left(y-y_{0}\right)^{2}+r^{2}=0,
$$

where $\left(x_{0}, y_{0}\right)$ is a hypothetical center of a circle.

In order to maximize accuracy, the approach to cavity localization is performed separately for each color channel. Our test results (as discussed in Section 4) suggest that the computational overhead introduced by this measure is acceptable in most application scenarios. In order to further lower computational demands, a limitation to one channel is possible. Based on the finding that histogram thresholding works best in the red channel, this channel is a prime candidate in application scenarios that require restriction to a single channel.

In the expermintal setup, we use the red, green and blue channel to perform edge detection. On every single image an edge detection filter is performed. To get an optimized edge image, all three edge pictures are summed up to one. Based on the generated edge image, a Hough circle transform with fixed radius is computed. After this transformation the Hough image is searched for the pixel with the highest intensity. This pixel indicates the center of the well and the coordinates can be extracted.

Based on the coordinates and the knowledge of the diameter of the cavity, a binary mask is created, which is used to eliminate irrelevant information surrounding the well.

\subsection{Liquid Detection by Thresholding}

A global threshold $T$ is used to distinguish between filled and empty cavities. For each cavity $i$, thresholding is performed on the average intensity of the red channel $r(i)$ of the region-of-interest. The filling status $f(i)$ is given as

$$
f(i)= \begin{cases}0 & \text { if } r(i)>T \\ 1 & \text { if } r(i) \leq T\end{cases}
$$

where $f(i)=0$ denotes a filled cavity at position $i$ and $f(i)=1$ denotes an empty cavity at position $i$.
$T$ is determined in an initial tuning phase. Depending on the exact lighting conditions and the absorption properties of liquid at hand, the values of an ideal setting for $T$ will differ. We have found that the classes in the proposed setup are well distinguished. Therefore, $T$ can be easily determined, e.g. by using an adaption of the iterative method proposed by [5].

1. Select an initial estimate for global threshold $T$.

2. Segment the image using $T$. This will produce two groups of wells: $G_{1}$ consisting of all wells with average intensity values $>T$, and $G_{2}$ consisting of all wells with intensity values $\leq T$.

3. Compute the average intensity values $m_{1}$ and $m_{2}$ for the wells in $G_{1}$ and $G_{2}$, respectively.

4. Compute a new threshold value: $T=\frac{1}{2}\left(m_{1}+m_{2}\right)$

5. Repeat steps 2 through 4 until the difference between values of $T$ in successive iterations is smaller than a predefined parameter $\Delta T$.

\section{Experimental Results}

To capture the images a standard Delock Industry USB 95852 camera module is mounted on top of a flat-bottom MTP96 microplate. For illumination, a commercial 950nm LED was used. To guarantee a precise positioning for every cavity between the camera and the LED, the plate transport of an Infinite F200 monochromator from Tecan Austria $\mathrm{GmbH}$ was used. This plate transport has a step size of one micrometer.

The measurements were performed using a volume of $200 \mu \mathrm{l}$. Successful differentiation between full and empty was also achieved using volumes of $50 \mu \mathrm{l}$ and water droplets. Figure 3(a) shows the results for unprocessed images. Figure 3(b) shows the results for images processed according to the method described above.

As can be seen, without preprocessing no simple threshold can be found and the aformentioned simplistic approach to liquid detection fails. 


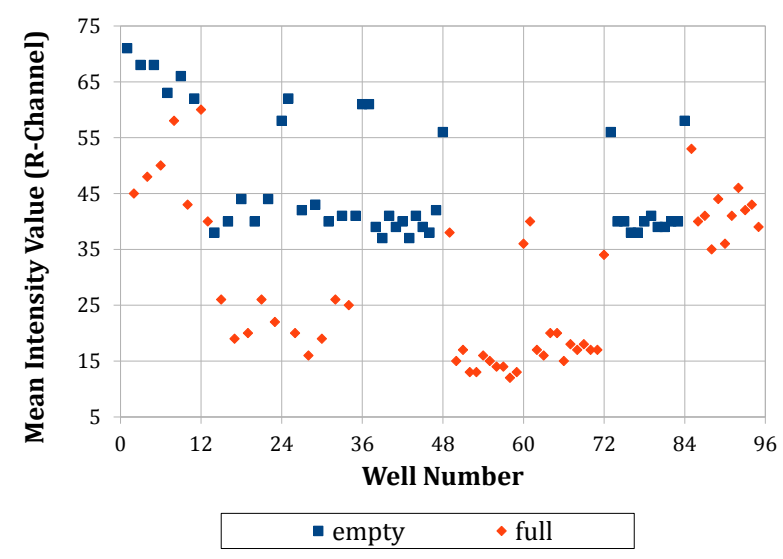

(a) Unprocesssed Images

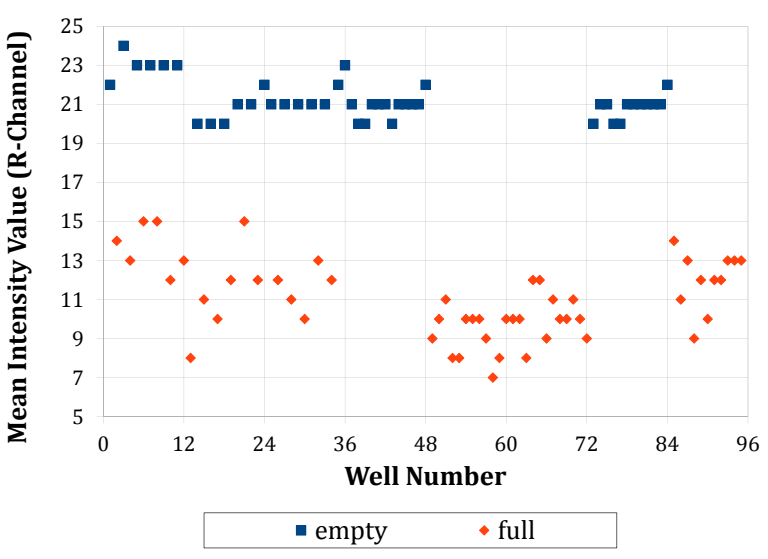

(b) Processed Images

Figure 3. Experimental Results

With preprocessing, the classes are clearly separated and the threshold can easily be chosen: The iterative method mentioned above used with $\Delta T=1$ and an initial threshold of 0 converges after three iterations.

Using a single-threaded Java implementation with batch processing on a Intel Pentium Dual CPU T2390 with 1.86 $\mathrm{GHz}$ and 3GB of RAM running on an Oracle JVM Version 6 on 32-bit Windows 7, the proposed approach had an average analysis time of 634 milliseconds per picture.

\section{Conclusion}

Initial evaluation shows that the proposed method reliably detects liquid in microplate cavities using comparatively inexpensive components. Compared to manual inspection, this is a significant advantage.

The evaluation performed so far has to be rated as a proof of concept that shows the feasibility of the proposed approach. Further testing and evaluation in a variety of lab conditions is necessary to assess the applicability of the approach in a more extensive scenario.

A step beyond classification of absence/presence of liquid is the reliable detection of the amount of water in the well. However, our experiments showed that with the used approach no accurate correlation between average intensity value and filling level coud be achieved.

Future work will focus on tuning the method and investigating possibilities to enhance the method from a binary classification of presence/absence to detecting the actual level of filling.

\section{References}

[1] O. Chapelle, P. Haffner, and V. Vapnik. Support vector machines for histogram-based image classification. IEEE
Transactions on Neural Networks, 10(5):1055-1065, 1999.

[2] D \& A Instrument Company. Light absorption \& scattering in water samples, 2005. [Online; Retrieved April 25, 2011].

[3] T. K. Ganga and V. Karthikeyani. Medical image segmentation using multi resolution histogram. In Proc. $3 r d$ Int Electronics Computer Technology (ICECT) Conf, volume 6, pages 267-269, 2011.

[4] L. Gil-Sanchez, E. Garcia-Breijo, J. Garrigues, N. Laguarda, R. Masot, J. Ibanez, J. Atkinson, and M. Glanc. Embedded pattern recognition systems for liquids classification: A comparison study. In Proc. IEEE Sensors, pages 1720-1723, 2011.

[5] R. C. Gonzalez and R. E. Woods. Digital Image Processing. Pearson Education, $3^{\text {rd }}$ edition, 2008.

[6] T. S. Newman and A. K. Jain. A survey of automated visual inspection. Computer Vision and Image Understanding, 61(2):231 - 262, 1995.

[7] H.-F. Ng. Automatic thresholding for defect detection. Pattern Recognition Letters, 27(14):1644-1649, Oct. 2006.

[8] K. J. Pithadiya, C. K. Modi, and J. D. Chauhan. Comparison of optimal edge detection algorithms for liquid level inspection in bottles. In Proc. 2nd Int Emerging Trends in Engineering and Technology (ICETET) Conf, pages 447-452, 2009.

[9] D. P. Seliskar, R. G. Waterbury, and R. E. Kearney. Proportional microvolume capacitive liquid level sensor array. In Proceedings fo the 2005 IEEE Engineering in Medicine and Biology $27^{\text {th }}$ Annual Conference, pages 7258-7261, Shanghai, China, Sept. 2005.

[10] M. Sezgin and B. Sankur. Survey over image thresholding techniques and quantitative performance evaluation. Journal of Electronic Imaging, 13(1):146-168, Jan. 2004.

[11] S. Siggelkow and H. Burkhardt. Improvement of histogrambased image retrieval and classification. In Proc. 16th Int Pattern Recognition Conf, volume 3, pages 367-370.

[12] L. Yazdi, A. S. Prabuwono, and E. Golkar. Feature extraction algorithm for fill level and cap inspection in bottling machine. In Proc. Int Pattern Analysis and Intelligent Robotics (ICPAIR) Conf, volume 1, pages 47-52, 2011. 